\title{
CALCULO DEL RIESGO DE ADVERSIDADES CLIMATICAS PARA LOS CULTIVOS: LOS CEREALES DE VERANO EN MONEGROS
}

\author{
Alberto MARTI EZPELETA \\ Dpto. de Geografía y Ordenación del Territorio \\ Universidad de Zaragoza
}

\begin{abstract}
Resumen: El mayor o menor riesgo de que ocurran adversidades climáticas en un territorio determina la rentabilidad y la posibilidad de establecimiento en éste de diversas especies y variedades de cultivo. Este trabajo presenta un método para calcular el riesgo de que se produzcan fenómenos climáticos adversos a lo largo del ciclo vegetativo de los cultivos, poniendo como ejemplo el caso de los cereales de verano en Monegros. El método se basa en la valoración del grado de vulnerabilidad del cultivo ante ciertas variables climáticas y de la probabilidad de ocurrencia de dichas variables.
\end{abstract}

Palabras clave: Adversidad climática, vulnerabilidad, riesgo de ocurrencia, maiz, arroz, ciclo vegetativo, Monegros.

\begin{abstract}
The bigger or the lesser danger that happen climatic adversities on a region will determine the profitability and the possibility of growth for several crops on that region. This work presents one method to calculate the risk of unfavourable climatic occurrences during the crop growing. The summer cereals at the Monegros region are one example. The method is based at the valuation of the crop vulnerability grade to some climatic phenomenon and the occurrence probability of these phenomenon.
\end{abstract}

Key words: Climatic adversity, vulnerability, occurrence risk, corn, rice, vegetal cycle, Monegros.

\section{INTRODUCCION}

El objetivo principal del agricultor es obtener el máximo y mejor producto con el menor trabajo y riesgo posible. Para poder alcanzar dicho objetivo es necesario adaptar los cultivos a las condiciones del clima de un territorio, seleccionando las 
especies y variedades más adecuadas que mejor resistan o eviten las adversidades climáticas. Llevar a cabo esta adaptación cultural exige, en primer lugar, conocer con detalle las condiciones ambientales de ese territorio y, en segundo lugar, la reacción de las plantas ante las condiciones desfavorables del clima a lo largo de su ciclo vegetativo.

En las siguientes páginas vamos a desarrollar un método de cálculo del riesgo de adversidades climáticas al que está sometido un cultivo en un territorio durante su desarrollo. El esquema metodológico empleado tiene como referencia los modelos de base ecológica para la planificación física que presentan Ramos Fernandez et. al. (1979) y especialmente el método de Ramos y Ayuso (1974) para la planificación de áreas rurales, si bien los parámetros que ellos utilizan son distintos a los utilizados en este estudio. Tras el apartado en el que se presenta la metodología utilizada, ésta se aplica como ejemplo a los cereales de verano (maiz y arroz) en tres localidades de la comarca de los Monegros (Marti, 1993), donde presentan una importante tendencia a aumentar su superficie debido a la extensión del regadío.

Precipitacion

Bujaraloz

Ontiñena

Sariñena
$1950-90$

$1969-90$

$1960-87$
Temperatura

1963-80

$1968-90$

$1961-90$
Viento

$1970-80$

$1970-80$

$1970-80$

\section{METODOLOGIA}

El método utilizado se basa en la expresión de las relaciones entre los cultivos y los parámetros adversos más significativos del clima de la comarca para el desarrollo de las plantas, viendo su efecto individual frente a cada uno de ellos a lo largo de su ciclo de desarrollo.

Para cuantificar estas relaciones agroclimáticas, que son las que en mayor medida limitan y condicionan el desarrollo de una planta en un territorio dado, calcularemos el riesgo de ocurrencia de adversidades climáticas al que están expuestos los cereales de verano en la zona de estudio. Consideramos que el riesgo de adversidad viene dado por la concurrencia de dos parámetros:

- el grado de vulnerabilidad que el cultivo presenta ante ciertos fenómenos climáticos a lo largo de su ciclo de desarrollo y

- la probabilidad de ocurrencia de estos fenómenos en el territorio analizado. 
Para definir el riesgo de adversidad al que están expuestos los cultivos tendremos que seleccionar las variables climáticas que son claves para la actividad agrícola, es decir, aquellos elementos y sus valores correspondientes que condicionan y limitan la implantación del cultivo y su desarrollo. Las variables que hemos utilizado en nuestro análisis han sido: temperaturas elevadas, temperaturas bajas, precipitaciones intensas y vientos fuertes. El déficit hídrico no se ha considerado ya que los cultivos analizados se desarrollan en regadío y ven cubierta siempre su demanda de agua a lo largo de todo el ciclo vegetativo.

En el caso de las precipitaciones intensas y de los vientos fuertes los valores umbrales elegidos han sido los mismos para los dos cultivos y durante todo el ciclo de desarrollo. Sin embargo, los umbrales térmicos, por debajo o por encima de los cuales las temperaturas registradas eran perjudiciales para las plantas, varían en función del tipo de cultivo y del momento del ciclo vegetativo.

Una vez seleccionadas las variables climáticas es preciso valorar de alguna manera el grado de vulnerabilidad de los cultivos ante dichas variables. Para ello hemos escogido una escala numérica del 0 al 3 con la que definiremos cada una de las décadas (período de diez días) que compone el ciclo de los cultivos y para cada una de las variables climáticas. El resultado será una matríz numérica en la que quedarán expresados los grados de vulnerabilidad que ese cultivo presenta durante todo su desarrollo, según la siguiente valoración y criterios:

- La vulnerabilidad será 0 cuando la variable climática correspondiente no afecte en ningún sentido al cultivo.

- El valor será 1 cuando los efectos que pueda producir dicha variable a la planta sean leves y sólo provoquen retrasos en el crecimiento o pequeñas pérdidas del rendimiento.

- Con una vulnerabilidad media, 2, se señalan aquellos períodos en los que los factores climáticos pueden representar un riesgo moderado sobre el desarrollo de los cultivos.

- Finalmente el valor 3 se asignará a las décadas en las que la acción del clima pueda tener como consecuencia una fuerte reducción del rendimiento del cultivo o, incluso, la muerte parcial o total de la planta.

La cuantificación de la probabilidad de ocurrencia de los fenómenos climáticos considerados se obtiene del análisis de los elementos climáticos que indica la frecuencia de aparición de dichos fenómenos en distintos puntos de la comarca. Esta 
probabilidad de ocurrencia la hemos valorado también según una escala del 0 al 3 , desde inexistente hasta alta.

La asignación de estos valores a las variables climáticas estará en función de la mayor o menor frecuencia de que se registren en cada una de las décadas. Los intervalos que definen esta frecuencia variarán con cada una de las variables, como veremos más adelante.

Una vez cuantificados el grado de vulnerabilidad de los cultivos y el riesgo de ocurrencia de las variables climáticas que pueden alterar negativamente su desarrollo podremos calcular el riesgo de adversidades climáticas al que están expuestos dichos cultivos en ocho localidades de Monegros. Para ello multiplicaremos las matrices con los valores de vulnerabilidad de los cultivos por cada una de las matrices que representan la probabilidad de ocurrencia de los fenómenos climáticos, obteniendo así nuevas matrices para cada cultivo en las que aparecerán unos valores numéricos comprendidos entre 0 y 9 correspondientes a los distintos grados de riesgo de adversidad:

0 Inexistente vulnerabilidad nula y probabilidad inexistente vulnerabilidad nula y probabilidad baja, media o alta vulnerabilidad baja, media o alta y probabilidad nula

1 Muy bajo vulnerabilidad baja y probabilidad baja

2 Bajo vulnerabilidad baja y probabilidad media vulnerabilidad media y probabilidad baja

3-4 Medio vulnerabilidad alta y probabilidad baja vulnerabilidad baja y probabilidad alta vulnerabilidad media y probabilidad media

6 Alto vulnerabilidad media y probabilidad alta vulnerabilidad alta y probabilidad media

9 Muy alto vulnerabilidad alta y probabilidad alta 

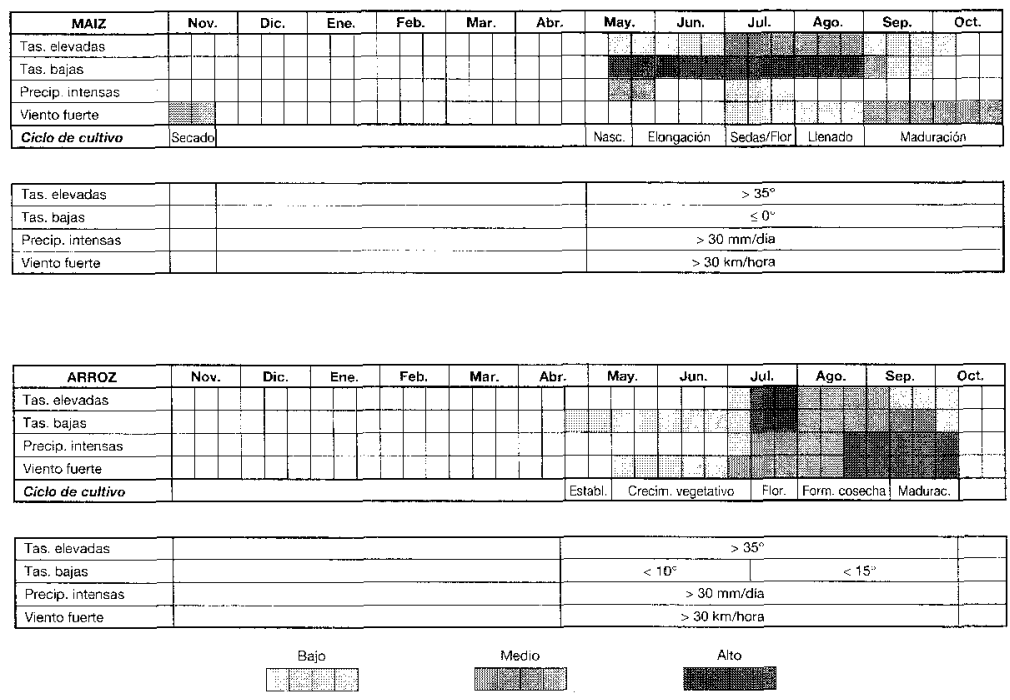

Figura 1.- Grado de vulnerabilidad de los cultivos a algunas variables climáticas.
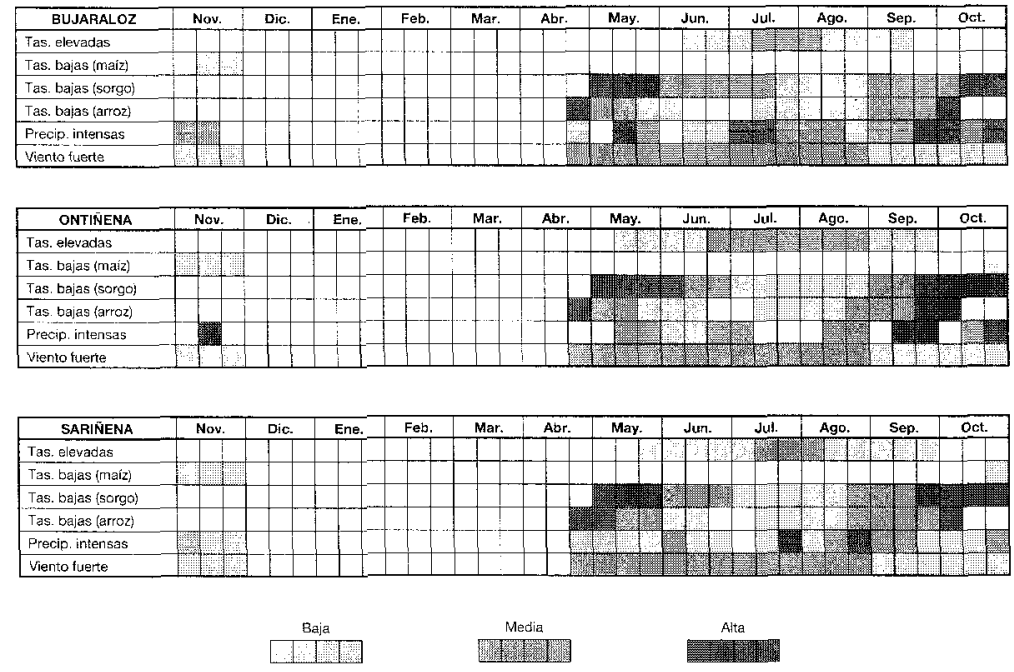

Figura 2.- Probabilidad de ocurrencia de los valores climáticos adversos para cereales de verano. 


\section{GRADO DE VULNERABILIDAD DE LOS CULTIVOS}

Los gráficos de la Figura 1 muestran los momentos, a lo largo del ciclo de desarrollo de los cereales de verano, en los que éstos presentan una mayor o menor vulnerabilidad a las variables climáticas adversas. Vienen constatados además los valores umbrales de estas variables que se han utilizado para determinar el grado de vulnerabilidad de estos cultivos. Como veremos, los períodos que coinciden con la floración, la formación de la cosecha y la madurez son los más sensibles ante la acción de las variables climáticas extremas.

El maíz.- La vulnerabilidad del maíz a las temperaturas elevadas es en general baja; con valores superiores a $35^{\circ}$ el crecimiento del maíz se detiene. Es durante la floración cuando puede sufrir más daño pues temperaturas extremas en este período incrementa el número de plantas estériles y disminuye el número de granos por mazorca. Normalmente no se producen asurados del maíz debidos a altos valores térmicos, a no ser que éstos coincidan con una escasez de agua en el suelo al haber transcurrido bastantes días desde el último riego.

Es un cultivo muy vulnerable a las heladas desde la germinación de las plántulas hasta el inicio del secado de los granos. Las heladas pueden causar la muerte de las plantas jóvenes. A partir de este momento, y hasta el final del ciclo, el frío y sobre todo las heladas ayudan a secar más el grano.

A partir de la siembra y hasta la aparición de las plántulas en la superficie una precipitación intensa puede producir el encarado o encostramiento de la capa superficial del suelo, dificultando en gran medida la nascencia del maíz, por lo que señalaremos con vulnerabilidad alta hacia esta variable los primeros días del ciclo. Durante el resto del ciclo de cultivo no se suelen producir otros problemas debidos a las precipitaciones intensas. Sólo durante la floración éstas pueden dificultar en parte la fecundación. Es más perjudicial una humedad elevada y contínua causada por días de precipitación consecutivos.

El viento puede afectar algo durante la floración pero puede ser más dañino durante la maduración del cultivo, especialmente cuando éste está enfermo debido a que el tallo queda hueco y es muy vulnerable a romperse por el viento.

El arroz.- La vulnerabilidad del arroz ante valores térmicos elevados $\left(>35^{\circ}\right)$ es alta durante la floración ya que pueden provocar la esterilidad de las flores. También son desfavorables, aunque en menor grado, durante la formación de la cosecha y la maduración de los granos. 
Del mismo modo las temperaturas inferiores a $10^{\circ}$ provocan la parada de la actividad vegetativa a lo largo del crecimiento de las plántulas. En la floración unos valores térmicos por debajo de $15^{\circ}$ pueden inducir la esterilidad de las flores, mientras que durante la formación de la cosecha y la maduración estos valores son desfavorables para la semilla ya que disminuye su peso total.

Las precipitaciones intensas y el viento fuerte constituyen dos factores negativos para el desarrollo del cultivo, especialmente en el período final en el que pueden producir el encamado y una importante descenso del rendimiento. La floración puede verse asímismo dificultada por la lluvia y el viento. Es por ello por lo que la vulnerabilidad es media y alta desde la floración del cultivo hasta su recolección.

\section{PROBABILIDAD DE OCURRENCIA DE LOS FENOMENOS CLIMATICOS}

Los grados de frecuencia correspondientes a los valores umbrales elegidos en el caso de las temperaturas máximas y mínimas, las precipitaciones intensas, el viento fuerte y el déficit hídrico los vamos a agrupar en cuatro grupos (del 0 al 3) (Fig. 2) en función de los siguientes criterios:

\section{-1) Temperaturas elevadas y temperaturas bajas}

Valor umbral: varían en función del cultivo y de la etapa de desarrollo.

\begin{tabular}{ccc}
$\begin{array}{l}\text { Frecuencia decádica } \\
\text { de registro }\end{array}$ & $\begin{array}{c}\text { Probabilidad } \\
\text { de ocurrencia }\end{array}$ & $\begin{array}{c}\text { Valor } \\
\text { asignado }\end{array}$ \\
\hline $0 \%$ & inexistente & 0 \\
$0,1 \%-29,9 \%$ & baja & 1 \\
$30 \%-69,9 \%$ & media & 2 \\
$\geq 70 \%$ & alta & 3
\end{tabular}

\section{-2) Precipitaciones intensas}

Valor umbral: precipitaciones superiores a $30 \mathrm{~mm} /$ día. 


\begin{tabular}{ccc}
$\begin{array}{l}\text { Frecuencia decádica } \\
\text { de registro }\end{array}$ & $\begin{array}{c}\text { Probabilidad } \\
\text { de ocurrencia }\end{array}$ & $\begin{array}{c}\text { Valor } \\
\text { asignado }\end{array}$ \\
\hline $0 \%$ & inexistente & 0 \\
$0,1 \%-4,9 \%$ & baja & 1 \\
$5 \%-9,9 \%$ & media & 2 \\
$\geq 10 \%$ & alta & 3
\end{tabular}

\section{-3) Viento fuerte}

Valor umbral: vientos superiores a $30 \mathrm{~km} /$ hora.

\begin{tabular}{ccc}
$\begin{array}{c}\text { Frecuencia mensual } \\
\text { de registro }\end{array}$ & $\begin{array}{c}\text { Probabilidad } \\
\text { de ocurrencia }\end{array}$ & $\begin{array}{c}\text { Valor } \\
\text { asignado }\end{array}$ \\
\hline $0 \%$ & inexistente & 0 \\
$0,1 \%-19,9 \%$ & baja & 1 \\
$20 \%-29,9 \%$ & media & 2 \\
$\geq 30 \%$ & alta & 3
\end{tabular}

\section{RIESGO DE ADVERSIDADES CLIMATICAS}

Las Figuras 3 y 4 reflejan los distintos niveles de riesgo de adversidad que presentan los cultivos en tres localidades de Monegros, obtenidos de la combinación del grado de vulnerabilidad de las plantas ante la acción de algunas variables climáticas y la probabilidad de ocurrencia de éstas.

El maíz.- Para el cultivo del maíz el mayor riesgo de adversidad viene dado por las temperaturas elevadas (Fig. 3). Estas constituyen un riesgo entre muy bajo y medio durante buena parte de su ciclo de desarrollo, excepto en el último mes. Es en las décadas de julio y agosto cuando los niveles de riesgo son mayores pudiendo traducirse en una parada de la actividad vegetativa de la planta.

El viento fuerte constituye en toda la comarca un riesgo bajo desde la aparición de las sedas y la floración hasta el momento de la cosecha. 

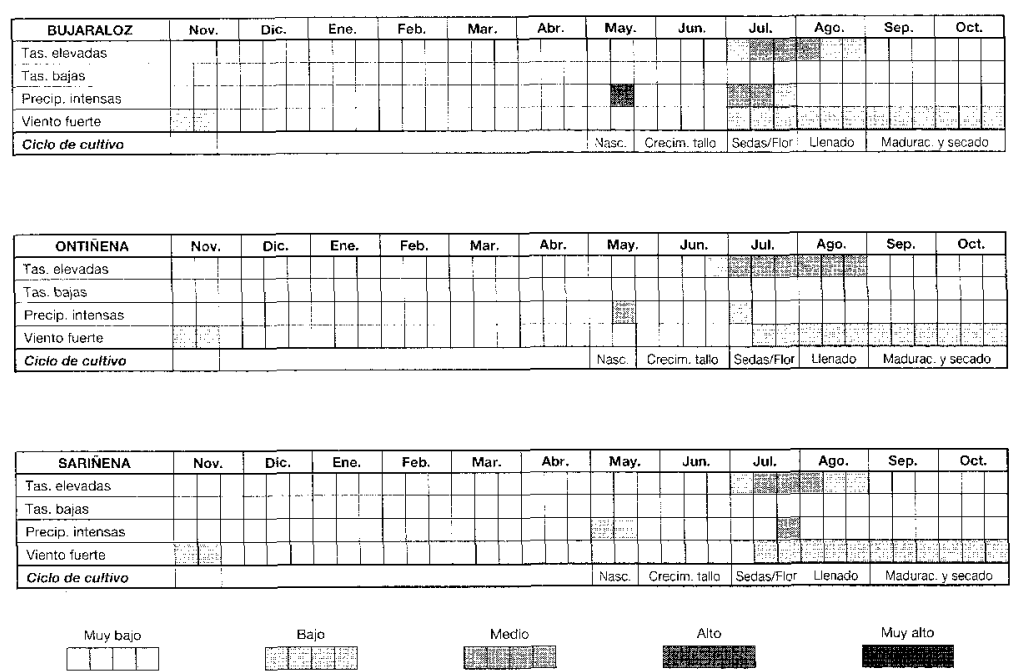

Figura 3.- Riesgo de adversidades climáticas del maiz en los Monegros.
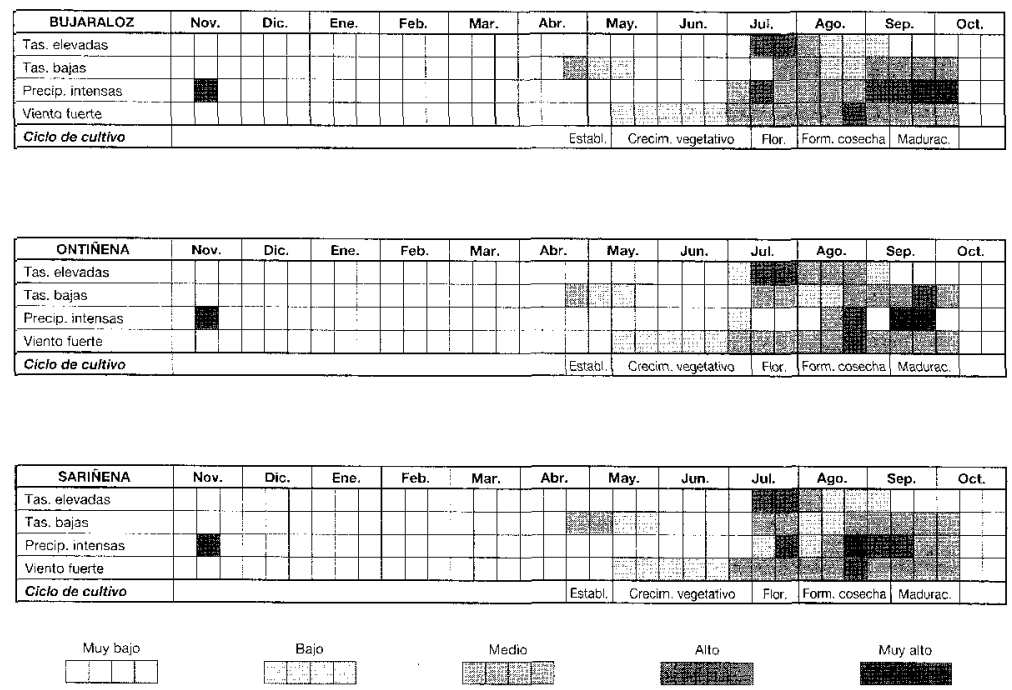

Figura 4.- Riesgo de adversidades climáticas del arroz en los Monegros. 
Menor es el riesgo de adversidad motivado por las precipitaciones intensas. En Bujaraloz y Ontiñena exíste un riesgo medio y alto durante los días posteriores a la siembra. También durante la floración se observan momentos en los que el riesgo de adversidad está entre muy bajo y medio, siendo inexistente durante el resto del ciclo de desarrollo.

Las temperaturas bajas no parecen constituir un riesgo para el crecimiento del maíz en los Monegros.

El arroz.- Este cultivo es afectado por las adversidades climáticas en mayor medida que el maíz (Fig. 4). Las temperaturas elevadas implican un riesgo de adversidad medio y alto entre mediados de julio y mediados de agosto, período en el que tiene lugar la floración y el comienzo de la formación de la cosecha. Durante el resto del ciclo el riesgo es bajo o inexistente.

Más continuado es el riesgo de adversidad motivado por temperaturas bajas, inferiores a $10^{\circ}$ y $15^{\circ}$, que alcanza niveles medios desde la floración hasta la maduración de los granos, llegando a ser altos en alguna década en Ontiñena.

El riesgo de adversidad por precipitaciones intensas comienza a partir de la floración, con niveles bajos y medios, llegando a ser durante la maduración alto y muy alto.

El viento fuerte conlleva para el cultivo del arroz un riesgo bajo y medio durante todo su desarrollo, bien sea dificultando la polinización o provocando el encamado. El riesgo es mayor en las décadas de julio y agosto, llegando a alcanzar un nivel alto en la. última década de agosto durante la formación de la cosecha.

\section{BIBLIOGRAFIA}

BARTOLINI, R. (1990): El maíz, Mundi-Prensa, Madrid.

LLANOS, M. (1984): El maiz: su cultivo y aprovechamiento, Mundi-Prensa, Madrid.

MARTI EZPELETA, A. (1993): Agroclimatología de los Monegros: estudio de aptitud agrícola en función de los condicionantes climáticos, Tesis Doctoral, Dpto. de Geografía. Universidad de Zaragoza. (Inédita).

PANDOLFI, A.M. (1988): "Vocazione colturale delle aree agrarie su base agroclimatica", Boll. Geof. VI, Agrimeteorologia. 
RAMOS, A. y AYUSO, E. (1974): "El medio ambiente natural. Un esquema metodológico para la planificación de áreas rurales", Bol. Est. Cent. Ecol, 3, 6, 19-25.

RAMOS, A. et al. (1979): Planificación física y Ecología. Modelos y métodos, Biblioteca Universitaria, E.M.E.S.A., Madrid.

TOlOSA ZUMETA, M. (1989): "El arroz en Aragón", Surcos de Aragón, 14, 27-31, Dpto. Agricultura, Ganadería y Montes de la Diputación General de Aragón. 